Reprod. Nutr. Dévelop., 1980, 20 (5 B), 1679-1684.

\title{
Contribution à l'étude de la répartition des espèces de Bifidobacterium dans la flore fécale de nourrissons alimentés soit au sein, soit par des laits maternisés
}

par Christel NEUT, Ch. ROMOND, H. BEERENS

Microbiologie, Unité d'Enseignement ef de Recherche de Pharmacie

3, rue Laguesse, 59045 Lille Cedex, France.

Summary. A contribution to the study of the distribution of Bifidobacterium species in the fecal flora of breast-fed and bottle-fed babies.

232 Bifidobacterium strains were isolated from the fecal samples of 50 infants. The species $B$. bifidum was predominant in breast-fed infants, while B. longum was most frequently isolated from infants receiving formulas.

\section{Introduction.}

L'alimentation du nouveau-né influence les conditions physico-chimiques ef microbiologiques de l'intestin. L'allaitement maternel provoque des selles liquides à «odeur de fromage » et à $\mathrm{pH}$ acide (environ 5,0) (Bullen et Willis, 1971). Les selles de l'enfant nourri artificiellement ressemblent à celles d'adultes. Elles sont de consistance ferme à odeur fécale et à $\mathrm{pH}$ compris entre 6,0 et 7,0 .

L'examen microbiologique montre, chez l'enfant nourri au sein, une prédominance de Bifidobacterium à un niveau de $10^{9} \mathrm{germes} / \mathrm{g}$ de matières fécales (Bullen ef al., 1976). La colonisation par des germes de putréfaction (Bacteroides, Clostridium) et des coliformes a lieu à partir de la $2^{\mathrm{e}}$ semaine mais ces espèces restentà des niveaux bas.

Chez l'enfant nourri artificiellement, les Bifidobacterium sont également présents mais leur taux est inférieur à celui observé chez l'enfant nourri au sein. La différence primordiale consiste en la présence de Bacteroides, Clostridium ef E. coli à des taux élevés. Sous allaitement maternel, l'enfant se défend contre l'invasion de son intestin par des E. coli, Bacteroides et Clostridium ce qui lui confère une résistance accrue aux gastroentérites. Deux hypothèses sont possibles :

- Soit les Bifidobacterium ne sont que les témoins de bonne santé de l'intestin, soit ils participent à l'établissement et au maintien de cette bonne santé. Les défenseurs de la première hypothèse ont invoqué la présence, dans le colostrum humain, de substances inhibitrices d'E. coli (Bullen, Tearle et Stewart, 1977) empêchant l'implantation de ce germe mais favorisant l'installation des bifidobactéries. Ces dernières acidifient fortement'le milieu par production d'acide acétique, produit métabolique majeur. Le 
pH du milieu intestinal s'abaisse et, compte tenu, du faible pouvoir tampon du lait maternel, il reste acide $(\mathrm{pH} 5,0)$ et inhibe la prolifération des Bacteroides, Clostridium ef $E$. coli. Le maintien de cet équilibre est assuré par les caractéristiques physicochimiques du lait maternel (concentration élevée en lactose, substrat pour les bifidobactéries ; faible concentration en protéines, substrat des germes de putréfaction et enfin faible pouvoir tampon). Les laits «maternisés » tentent de reproduire les propriétés physico-chimiques du lait maternel mais sont incapables de maintenir la flore bifide de l'enfant nourri au lait maternel. On peut en conclure que les seules caractéristiques physico-chimiques évoquées plus haut ne suffisent pas à maintenir la flore bifide. On peut alors penser que le lait maternel apporte, en plus, des facteurs indispensables au développement intestinal des Bifidobacterium.

- La deuxième hypothèse envisage une participation active des bifidobactéries, les mêmes facteurs étant à l'origine de leur implantation. L'étude de ces facteurs a fait l'objet de très nombreuses recherches. Plusieurs substances présumées bifidigènes ont été décrites mais leur administration n'a jamais créé chez le nourrisson l'équilibre intestinal de l'allaitement maternel.

Le lactulose (le facteur Bifidus de Petuely (Gedek, 1968)) ne peut être responsable de l'effet spécifique du lait maternel car ce sucre n'est pas présent dans un lait non traité (Mendez et Olano, 1979). D'autres travaux (Famura ef al., 1977) ont identifié un dérivé de l'acide pantothénique isolé de la carotte comme facteur bifidigène pour une souche de Bifidobacterium infantis. Yazawa, Imai et Tamura (1978) ont suggéré l'utilisation de différents oligo- et polysaccharides comme agents sélectifs des bifidobactéries intestinales. Ils proposent le raffinose, le stachyose et l'inuline testés vis-àvis d'une seule souche de B. infantis et une de B. breve. Kehagias et al. (1977) ont isolé un produit d'hydrolyse de caséine bovine favorisant la croissance de $B$. bifidum variété pennsylvanicus. Des sucres aminés et des glycopeptides de faible poids moléculaire (facteur Bifidus II de Gyorgy) sont cités par différents auteurs comme bifidigènes (Gedek, 1968).

Les facteurs bifidigènes décrits semblent spécifiques de l'espèce étudiée (Neut et al., 1980). Notre but est de reproduire dans l'intestin du nourrisson l'équilibre de flore constaté chez l'enfant alimenté au lait de femme. Les questions que nous nous posons sont les suivantes : Existe-t-il une espèce de Bifidobacterium spécifique de l'intestin du nourrisson alimenté par sa mère, si oui quelle est-elle?

Beaucoup d'auteurs (Hewitt et Rigby, 1976 ; Hoogkamp-Korstanje ef al., 1979 ; Bullen, Tearle et Willis, 1976 ; Ellis-Pegler, Crabtree et Lambert, 1975 ; Mata et Urrutia, Patte et al., 1979) se sont intéressés simplement au genre Bifidobacterium mais peu (Mitsuoka, Hayakawa ef Kimura, 1974 ; Reuter, 1963 ; Dehnert, 1957) ont poussé leurs investigations jusqu'à la détermination des espèces. Notre travail consiste à rechercher et identifier les Bifidobacterium des matières fécales d'enfants nourris au lait maternel d'une part et aux laits artificiels d'autre part.

\section{Matériel et méthodes.}

Les matières fécales des enfants sont recueillies par écouvillonnage rectal sur culturettes anaérobies (Marion Scientific, Kansas-City, USA) pour éviter l'action 
toxique de l'oxygène de l'air sur les Bifidobacterium pendant le transport. 50 enfants sont retenus pour cette étude, ils sont tous âgés de plus d'une semaine, sont en bonne santé ou hospitalisés pour des pathologies diverses mais non digestives et aucun ne recevait d'antibiotiques, 11 sont nourris au sein et 39 reçoivent un allaitement artificiel.

Isolement ef identification des Bifidobacterium. - Les prélèvements de selles sont conservés en anaérobiose et sont examinés dans les six heures au plus tard. Une série de dilutions de raison 10 est préparée (jusqu'à $10^{-7}$ ) avec comme diluant une solution de Ringer au $1 / 4$ et cystéinée, $0,1 \mathrm{ml}$ des 4 dernières dilutions sont étalées sur un milieu VL solide (Buttiaux, Beerens ef Tacquet, 1974) additionné de 10 p. 100 de sang de cheval.

Après 5 jours d'incubation en anaérobiose à $37{ }^{\circ} \mathrm{C}$, tous les types de colonies et en moyenne la racine carrée du nombre présent à la dernière dilution sont réisolés pour purification et identifiés par leur morphologie et les caractères biochimiques décrits dans la 8 e édition de 1974 du Bergey's manual of determinative bacteriology. Dans une même selle les souches mentionnées sont différentes. Elles se caractérisent, en effet :

- soit par la morphologie des colonies,

- soit par l'utilisation ou non de substrats hydrocarbonés non spécifiques de la définition de l'espèce, par exemple le saccharose, le maltose pour B. bifidum.

\section{Résultats.}

Le tableau 1 considère la répartition de différentes espèces de Bifidobacterium, dans 11 selles de nourrissons alimentés au sein, on constate 5 fois l'association B. bifidum et $B$. longum, 5 fois $B$. bifidum seul (aux dilutions élevées) et 1 fois $B$. longum.

\section{TABLEAU 1}

Répartition des différentes espèces de Bifidobacterium dans les selles de nourrisson

\begin{tabular}{|c|c|c|}
\hline Allaitement & Association des espèces de Bifidobocterium par selle & $\begin{array}{c}\text { Nombre } \\
\text { de cas }\end{array}$ \\
\hline $\begin{array}{l}\text { Maternel } \\
11 \text { selles }\end{array}$ & 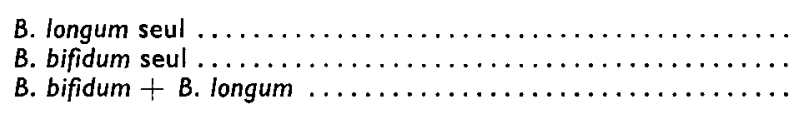 & $\begin{array}{l}1 \\
5 \\
5\end{array}$ \\
\hline $\begin{array}{l}\text { Artificiel } \\
39 \text { selles }\end{array}$ & 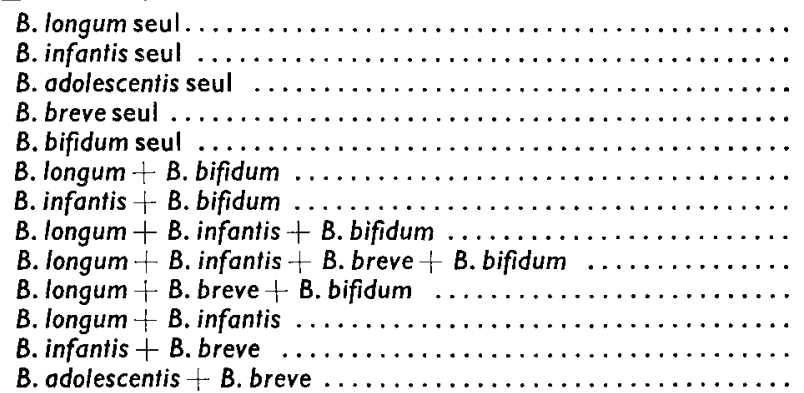 & $\begin{array}{r}14 \\
4 \\
1 \\
1 \\
1 \\
6 \\
1 \\
4 \\
1 \\
1 \\
2 \\
2 \\
1\end{array}$ \\
\hline
\end{tabular}


Sur 39 selles d'enfants alimentés avec différents laits maternisés, il existe une grande diversité de possibilités puisque les espèces rencontrées sont plus nombreuses.

Le tableau 2 détaille les 232 souches de Bifidobacterium isolées des 50 selles se répartissant en 5 espèces : $B$. longum (113), B. bifidum (77), B. infantis (28), B. breve (12) et $B$. adolescentis (2). Les 2 espèces de Bifidobacterium les plus importantes chez les nourrissons, toutes alimentations confondues, sont $B$. longum et $B$. bifidum. En séparant les 2 types d'alimentation, trois remarques sont possibles :

TABLEAU 2

Distribution des différentes espèces de Bifidobacterium suivant le type d'allaitement

\begin{tabular}{|c|c|c|c|c|}
\hline & & Total & $\begin{array}{l}\text { Allaitement } \\
\text { artificiel }\end{array}$ & $\begin{array}{l}\text { Allaitement } \\
\text { maternel }\end{array}$ \\
\hline \multicolumn{2}{|c|}{ Nombre de selles } & 50 & 39 & 11 \\
\hline \multicolumn{2}{|c|}{$\begin{array}{c}\text { Nombre total de souches de Bifido- } \\
\text { bacterium } \ldots \ldots \ldots \ldots \ldots \ldots \ldots \ldots\end{array}$} & 232 & 153 & 79 \\
\hline \multirow{2}{*}{ B. bifidum } & Souches. & 77 & 20 & 57 \\
\hline & p. $100 \ldots \ldots$ & 33 & 13 & 72 \\
\hline \multirow{2}{*}{ B. infantis } & Souches..... & 28 & 28 & 0 \\
\hline & p. 100 & 12 & 18 & 0 \\
\hline \multirow{2}{*}{ B. longum } & Souches..... & 113 & 91 & 22 \\
\hline & p. 100 & 48,7 & 60 & 28 \\
\hline \multirow{2}{*}{ B. adolescentis } & Souches.... & 2 & 2 & 0 \\
\hline & p. $100 \ldots \ldots$ & 0,8 & 1 & 0 \\
\hline \multirow{2}{*}{ B. breve } & Souches..... & 12 & 12 & 0 \\
\hline & p. $100 \ldots \ldots$ & 5 & 8 & 0 \\
\hline
\end{tabular}

1) Nous constatons dans une même selle l'existence de plusieurs souches d'une même espèce. Elles se différencient les unes des autres par des caractères mineurs.

2) 39 selles proviennent d'enfants allaités avec un laił maternisé, nous obtenons 153 souches de différentes espèces de Bifidobacterium B. longum (91 souches) est la plus fréquente suivie de très loin par $B$. infantis (28 souches) et B. bifidum (20). B. breve n'est isolé que 12 fois ef $B$. adolescentis 2 fois.

3) Dans les 11 selles de nourrissons alimentés au sein, nous recueillons 79 Bifidobacterium dont 57 sont de l'espèce $B$. bifidum. Les 22 autres souches appartiennent à $B$. longum et nous ne trouvons ni B. infantis, ni B. breve, ni B. adolescentis.

\section{Discussion.}

Nous n'utilisons pas, dans ce travail, de milieu sélectif afin de permettre le déve- 
loppement de toutes les souches de Bifidobacterium. De plus, nous ne nous adressons qu'aux espèces majoritaires présentes aux dilutions de taux élevé ce qui correspond bien au buł que nous nous sommes fixés.

Cette méthode fait apparaître une grande simplicité des résultats (2 espèces) dans l'alimentation maternelle par opposition à une plus grande complexité dans l'alimentation artificielle ( 5 espèces). On peut invoquer un nombre plus faible d'examens dans l'alimentation maternelle (11 contre 39). Ceci est la conséquence de difficultés techniques. Nous ne retenons que des enfants au sein depuis leur naissance et âgés de plus d'une semaine afin d'éviter les fluctuations de la flore dans les premiers jours mais ces cas sont difficiles à trouver.

Du tableau 1, il ressort que dans l'allaitement maternel le germe majoritaire est B. bifidum. Il est retrouvé, en effet, dans 10 selles sur 11 soit seul ( 5 fois sur 11 ) soit en association avec $B$. longum ( 5 fois sur 11 ). $B$. longum est isolé comme majoritaire 1 fois sur les 11 échantillons.

Dans le tableau 2, lorsqu'on s'adresse au nombre de souches isolées la même conclusion s'impose : $B$. bifidum représente 72 p. 100 des souches isolées dans l'allaitement maternel.

Lorsqu'on s'adresse à l'alimentation par laits maternisés, les résultats sont très différents. B. longum est le plus fréquent, il est isolé 27 fois sur les 39 prélèvements examinés. Il est seul, aux dilutions de taux élevé, 14 fois et en association avec d'autres Bifidobacterium 13 fois. D'autres espèces de Bifidobacterium sont retrouvées, quelquefois, comme majoritaires $B$. infantis $4 / 39, B$. adolescentis $1 / 39, B$. breve $1 / 39$ ef $B$. bifidum $1 / 39$. Dans cette deuxième série, $B$. bifidum est isolé 14 fois dans les 39 échantillons mais il est généralement en association avec d'autres Bifidobacterium. Si on compte le nombre de souches isolées (tabl. 2) dans les 39 selles, on constate là encore une majorité de B. longum (60 p. 100) alors que B. bifidum ne représente que 13 p. 100.

\section{Conclusion.}

On peut admettre que l'espèce de Bifidobacterium la plus fréquente dans l'intestin du nourrisson au sein est bien B. bifidum, d'autres travaux du laboratoire (Neut ef al., 1980) nous confortent dans cette idée. Par contre, $B$. longum semble globalement l'espèce majoritaire des selles d'enfants nourris artificiellement.

\section{Journées Ingestion-Digestion-Absorption de l'Association française de Nutrition, Paris, 15-16 novembre 1979.}

Remerciements. - Nous sommes très reconnaissants à Monsieur le Professeur Fontaine et à son équipe de l'aide qu'ils nous ont accordée fout au long de cette recherche.

\section{Références}

BERGEY D. H., 1974. Manual of determinative bacteriology, 1268, $8^{\mathrm{e}}$ éd. Williams and Wilkins Co., Baltimore.

BULLEN C. L., TEARLE P. V., STEWART M. G., 1977. The effect of « humanised » milks and supplemented breast feeding on the faecal flora of infants. J. Med. Microbiol., 10, 403-413. 
BULLEN C. L., TEARLE P. V., WILLIS A. T., 1976. Bifidobacteria in the intestinal tract of infants : an in vivo study. J. Med. Microbiol., 9, 325-333.

BULLEN C. L., WILLIS A. T., 1971. Resistance of the breast fed infant to gastroenteritis. Brit. Med. J., 3, 338-343.

BUTTIAUX R., BEERENS H., TACQUET A., 1974. Manuel de techniques bactériologiques, $4 \mathrm{e}$ éd., Flammarion Paris, 700 P.

DEHNERT J., 1957. Untersuchungen über die grampositive Stuhlflora des Brustmilchkindes. Z Zbl. Bact. I. Abt. Orig., 169, 66-86.

ELLIS-PEGLER R. B., CRABTREE C., LAMBERT H. P., 1975. The faecal flora of children in the United Kingdom. J. Hygiene, 75, 135-142.

FAMURA Z., NAKAJIMA T., SAMEJIMA K., YOSHLOKA M., NAKAMURA F., NAKAMURA H., INAGAKI M., 1972. Bifidus factor in carrot. $V$ : Isolation of new active substances. Proc. jap. med. Acad., 48, 138-143.

GEDEK B., 1968. Bakferiologische Faecesanalysen bei mit Milchaustauschern ernährten Kälbern. Zbl. Bokt. Paras. A, 209, 244-261.

HEWITT J. H., RIGBY J., 1976. Effect of various milk feeds on numbers of Escherichia coli and Bifidobacterium in the stools of new born infants. J. Hygiene, 77, 129-139.

HOOGKAMP-KORSTANJE J. A. A., LINDNER J. G. E. M., MARCELIS J. H., DEN DAAS-SLAGT H., DE VOS N. M., 1979. Composition and ecology of the human intestinal flora. Antonie van Leeuwenhoek, 45, 35-40.

KEHAGIAS C., JAO V. C., MIKOLAJCIK E. M., HANSEN P. M. T., 1977. Growth response of Bifidobacterium bifidum to a hydrolytic product isolated from bovine casein. J. Food Sci., 42, 146-150.

MATA L. J., URRUTIA J. J., 1971. Intestinal colonization of breast fed children in a rural area of low socio-economic level. Ann. N. J. Acad. Sci., 176, 93-98.

MENDEZ A., OLANO A., 1979. Lactulose, a review of some chemical properties and applications in infant nutrition and medecine. Dairy Sci. Abstr., 41, 531-535.

MISTUOKA T., HAYAKAWA K., KIMURA N., 1974. Die Faekalflora bei Menschen. II. Mitteilung : Die Zusammensetzung der Bifidobakterienflora der verschiedenen Altersgruppen. Z $\mathrm{bl}$. Bakt. I. Abt. Orig., 226, 469-478.

NEUT C., BEERENS H., ROMOND Ch., MONTREUIL J., 1980. Contribution à l'étude de la maternisation des laits : influence in vitro du lait maternel, du lait de vache et de laits maternisés sur la croissance des Bifidobacterium. Ann. Microbiol. (à paraître).

PATTE C., TANCREDE C., RAIBAUD P., DUCLUZEAU R., 1979. Premières élapes de la colonisation bactérienne du tube digestif du nouveau-né. Ann. Microbiol., 130, 69-81.

REUTER G., 1963. Vergleichende Untersuchungen über die Bifidus flora im Säuglings- und Erwachsenenstuhl. Zbl. Bakf. I. Abt. Orig., 191, 486-507.

YAZAWA K., IMAI K., TAMURA Z., 1978. Oligosaccharides and polysaccharides specifically utilizable by bifidobacteria. Chem. pharm. Bull. Tokyo, 26, 3306-3311. 